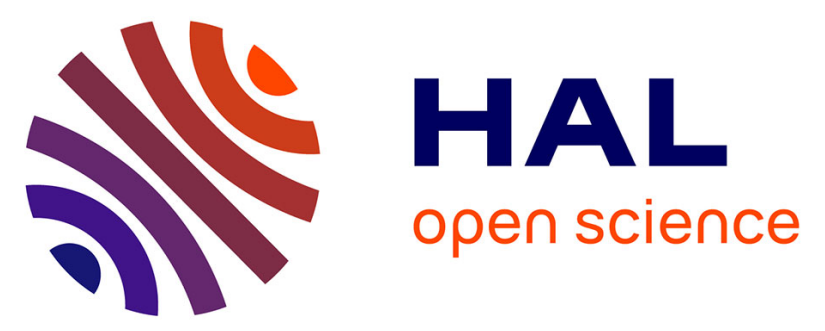

\title{
Induced magnetic fields and plasma motions in the inner part of the Martian magnetosphere
}

\author{
E. Dubinin, M. Fraenz, Ronan Modolo, Martin Pätzold, Silvia Tellmann, O. \\ Vaisberg, S. Shuvalov, L. Zelenyi, L. Chai, Y. Wei, et al.
}

\section{- To cite this version:}

E. Dubinin, M. Fraenz, Ronan Modolo, Martin Pätzold, Silvia Tellmann, et al.. Induced magnetic fields and plasma motions in the inner part of the Martian magnetosphere. Journal of Geophysical Research Space Physics, 2021, 126 (12), pp.e2021JA029542. 10.1029/2021JA029542 . insu-03475721

\author{
HAL Id: insu-03475721 \\ https://hal-insu.archives-ouvertes.fr/insu-03475721
}

Submitted on 7 Jan 2022

HAL is a multi-disciplinary open access archive for the deposit and dissemination of scientific research documents, whether they are published or not. The documents may come from teaching and research institutions in France or abroad, or from public or private research centers.
L'archive ouverte pluridisciplinaire HAL, est destinée au dépôt et à la diffusion de documents scientifiques de niveau recherche, publiés ou non, émanant des établissements d'enseignement et de recherche français ou étrangers, des laboratoires publics ou privés.

\section{(ㄷ)(1) $\$$}

Distributed under a Creative Commons Attribution - NonCommerciall 4.0 International 


\section{JGR Space Physics}

\section{RESEARCH ARTICLE 10.1029/2021JA029542}

Key Points:

- Draping of the interplanetary magnetic field around Mars penetrates deep to the ionosphere enveloping the planet and driving the ionosphere to the bulk motion

- Draping and motion of the ionospheric plasma is characterized by asymmetry by the direction of the motional electric field in solar wind

- Ion acceleration and extraction from the ionosphere is accompanied by a shift of the bulk ionosphere in the opposite direction

Correspondence to:

E. Dubinin,

dubinin@mps.mpg.de

\section{Citation:}

Dubinin, E., Fraenz, M., Modolo, R., Pätzold, M., Tellmann, S., Vaisberg, O., et al. (2021). Induced magnetic fields and plasma motions in the inner part of the Martian magnetosphere. Journal of Geophysical Research: Space Physics, 126, e2021JA029542. https://doi. org/10.1029/2021JA029542

Received 11 MAY 2021 Accepted 15 NOV 2021

Author Contributions:

Conceptualization: E. Dubinin Formal analysis: E. Dubinin Investigation: E. Dubinin, M. Fraenz, R. Modolo, M. Pätzold, S. Tellmann, O. Vaisberg, S. Shuvalov, L. Chai, Y. Wei, J. McFadden, G. DiBraccio, J. Espley Methodology: E. Dubinin Software: M. Fraenz

\section{Induced Magnetic Fields and Plasma Motions in the Inner Part of the Martian Magnetosphere}

\author{
E. Dubinin ${ }^{1}$ (D), M. Fraenz ${ }^{1}$ (D), R. Modolo² ${ }^{(D)}$, M. Pätzold 3 , S. Tellmann ${ }^{3}$, O. Vaisberg 4 (D),

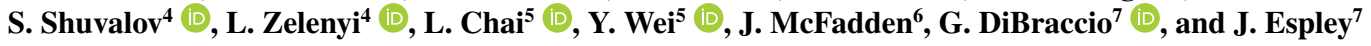 \\ ${ }^{1}$ Max-Planck-Institute for Solar System Research, Göttingen, Germany, ${ }^{2}$ Rheinisches Institut Fuer Umweltforschung, \\ Cologne, Germany, ${ }^{3}$ LATMOS/IPSL, UVSQ Universite, UPMC University Paris CNRS, Guyancourt, France, ${ }^{4}$ Institute of \\ Space Research, Moscow, Russia, ${ }^{5}$ Key Lab of Earth and Planetary Physics, Institute of Geology and Geophysics, Beijing, \\ People Republic of China, ${ }^{6}$ Space Sciences Laboratory, U. C. Berkeley, Berkeley, CA, USA, ${ }^{7}$ NASA Goddard Space Flight \\ Center, Greenbelt, MD, USA
}

Abstract Analysis of Mars Atmosphere and Volatile Evolution (MAVEN)/Supra-Thermal And Thermal Ion Composition observations in the Martian upper atmosphere, bounded at higher altitudes by the shocked solar wind, shows that the draping of interplanetary magnetic field penetrates down to low altitudes ( 200-250 km) and governs dynamics of the ionosphere. The upper ionospheric plasma is driven into motion flowing around Mars similar to the shocked solar wind in the adjacent magnetosheath. Such a fluid-like motion is accompanied by ion acceleration caused by the bending of the magnetic field, leading to ion extraction and finally to ion pickup. Extraction of ions and their acceleration produces a recoil effect of the bulk ionosphere in the opposite direction. This provides a strong asymmetry in ion dynamics in two different hemispheres, accompanied by wrapping of the magnetic field lines around Mars and respective reconnection.

Plain Language Summary Although the Martian magnetosphere is hybrid and contains components of the induced and intrinsic magnetosphere, is possible to display these components by using the specific coordinate systems. Here we study the properties of the induced magnetosphere using the data obtained by MAVEN spacecraft. The interplanetary magnetic field penetrates deep into the Martian ionosphere draping around Mars and drive to the motion dense ionospheric plasma. Draping features and the induced plasma motions occur different in two hemispheres determined by the direction of the motional electric field in the solar wind. Ion acceleration and extraction is accompanied by a recoil effect that leads to a shift and asymmetry of the ionosphere.

\section{Introduction}

The absence of a global magnetic field at Mars leads to nearly direct interaction of the solar wind with its atmosphere and ionosphere and to the formation of an induced magnetosphere where the interplanetary magnetic field (IMF) drapes around the ionospheric obstacle. In the case of a planet with an intrinsic magnetic field, the dayside plasma density is generally much lower than the density in the adjacent magnetosheath. By contrast, in the Martian case of an induced magnetosphere, the situation is different - the inner part of the induced magnetosphere of Mars or Venus is filled by much denser ionospheric plasma in which the dominant ion species are $\mathrm{O}_{2}^{+}$and $\mathrm{O}^{+}$ (Dubinin et al., 2011; Halekas et al., 2017; Nagy et al., 2004). An important feature of the induced magnetosphere of Mars is that the draping IMF penetrates into the ionosphere because the solar wind dynamic pressure generally exceeds the thermal plasma pressure in the upper Martian ionosphere (see e.g., Sanchez-Cano et al., 2020). A similar effect is observed at Venus during the periods of high solar wind pressure and/or during periods of low solar activity when the Venusian ionosphere becomes weaker (Luhmann et al., 1980; Russell \& Vaisberg, 1983).

At Mars, the existence of strong localized crustal magnetic fields (Acuña et al., 1999) significantly influences the interaction adding features typical for planets with a global intrinsic magnetic field. The topology of the magnetic field lines around these regions becomes very intricate, due to reconnection between the IMF and crustal fields (Hara et al., 2016; Harada et al., 2017, 2018, 2020; J. Halekas \& Brain, 2010; Xu et al., 2017, 2020). The net result might be the appearance of a twisted magneto tail (DiBraccio et al., 2018; Dubinin et al., 2017).

To separate features of the draping and crustal components of the Martian magnetosphere one can use different coordinate systems. The influence of the crustal field is rather complicated because of the intricate, localized 
topology of the crustal field lines coupled with Martian rotation. Nevertheless, one may expect a global effect of the crustal fields on the magnetic topology at Mars while considering a statistical field configuration by averaging over many Mars rotations and using the Mars Solar Orbital (MSO) coordinates in which the X-axis is directed from the center of Mars to Sun, the Z-axis is perpendicular to the planetary orbital plane and directed to the north, and Y completes the right-handed system (DiBraccio et al., 2018; Dubinin et al., 2017). The net field topology resembles topology of a hybrid magnetosphere with elements of the induced and intrinsic magnetospheres (Dubinin et al., 1980).

Since the orientation of the IMF varies with time, the relevant coordinate system for the description of the draping magnetosphere is the Mars Solar Electric System (MSE) which has the $X_{\mathrm{MSE}}$ axis antiparallel to the upstream solar wind flow (the solar wind aberration was not taken into account), the $Y_{\mathrm{MSE}}$ axis along the cross-flow magnetic field component of the IMF in the solar wind, and the $Z_{\mathrm{MSE}}$ axis pointing in the direction of the solar wind motional electric field ( $-V_{\mathrm{sw}} \times B_{\mathrm{IMF}}$ ), where $V_{s w}$ and $B_{\mathrm{IMF}}$ are the vectors of the velocity and the magnetic field in the solar wind, respectively (Dubinin et al., 1996; Moore et al., 1990; Russell et al., 1995; Yeroshenko et al., 1990). When we rotate the spacecraft trajectories into MSE coordinates and apply temporal averaging the effects of the crustal magnetic field on an asymmetry are significantly reduced. Figure 1 compares the values of the magnetic field in the MSE-reference frame in the $+Z_{\mathrm{MSE}}$ and $-Z_{\mathrm{MSE}}$ hemispheres (panels (a) and (d)) with the corresponding values in the $+Z_{\mathrm{MSO}}$ and $-Z_{\mathrm{MSO}}$ hemispheres of the MSO-frame (panels (b), (c), (e) and (f)). The magnetic field values in the MSO coordinates were plotted for $B_{\mathrm{ysw}} \geq 0$ and two cases of the Mars position. A reference point $(\mathrm{CF})$ on the Mars surface with the aerographic coordinates Lat $=-40^{\circ}$ and Long $=180^{\circ}$ characterized by the strong crustal sources of the magnetic field was near the noon $\left(0^{\circ}-30^{\circ}\right)$ and midnight meridians $\left(150^{\circ}-180^{\circ}\right)$, respectively. Comparing the data for $+Z_{\mathrm{MSE}}$ and $+Z_{\mathrm{MSO}}$ hemispheres we observe only a weak contribution of the crustal sources at low altitudes $(\leq 200 \mathrm{~km})$ at small and large solar zenith angles. Effects of the crustal fields clearly seen in the $-Z_{\text {MSO }}$ hemisphere (panels (e) and (f)) are strongly attenuated while plotting the data for the $-Z_{\text {MSE }}$ hemisphere that justifies our approach. Another biases might be related with the seasonal variations which can drive the important changes in the ionosphere dynamics. Their potential contribution is beyond the current study and must be tested in a future analysis.

In this investigation we focus on characterizing the main phenomenological features of the induced Martian inner magnetosphere, including the topology of the magnetic field as well as the induced ionospheric dynamics, and therefore we use MSE-coordinates. This is important because ultimately, this regime of interaction of the solar wind with the Martian upper atmosphere has important implications for the ionospheres global dynamics. It is a follow-up study of our previous work (Chai et al., 2019; Dubinin et al., 2019) with a particular focus on low altitude distributions of draped IMF and ionospheric plasma flows. The previous studies of Dong et al. (2015), Fang et al. (2010), Luhmann (1990) have suggested that the ion escape is driven through the induced electric field $E_{\mathrm{sw}}=-V_{\mathrm{sw}} \times B_{\mathrm{IMF}}$, resulting in an asymmetric outflow with the ion plume. However, the mechanisms of ion extraction from the ionosphere have not been fully investigated. The observations by Dubinin et al. (2019) showed that the ion escape at the nightside is driven through the ion trail filled by a dense ionospheric plasma and shifted to the hemisphere opposite to the hemisphere with the ion plume. Here we also investigate processes which lead to the formation of the ion trail.

\section{Instrumentation}

The Mars Atmosphere and Volatile Evolution (MAVEN) spacecraft arrived at Mars in September 2014 to study the processes in the upper atmosphere/ionosphere and its interaction with the solar wind (Jakosky et al., 2015). MAVEN was inserted into an elliptical orbit with periapsis and apoapsis of 150 and 6,200 km, respectively, and with a period of $4.5 \mathrm{hr}$. In this paper, we discuss observations made by the Supra-Thermal And Thermal Ion Composition (STATIC) instrument from November 2014 to May 2018. The STATIC instrument mounted on the actuated payload platform is used to study the dynamics of planetary ions. It measures energy spectra of ion fluxes in the range of $0.1 \mathrm{eV}-30 \mathrm{keV}$ and the ion composition (McFadden et al., 2015). The measurements allow a retrieval of the velocity distribution functions and their moments (density, velocity, temperature) for different ion species. The STATIC instrument samples all received time-of-flight events onboard with a resolution of 64 energy bins, 64 mass bins, 16x16 field of view bins, and 4s time resolution. Because the resulting matrix is far too big to be downloaded as a whole, only down-sampled 'products' are transmitted which represent sums over one or more dimensions of the original 5-dimensional matrix (McFadden et al., 2015). Not all products are 
a)
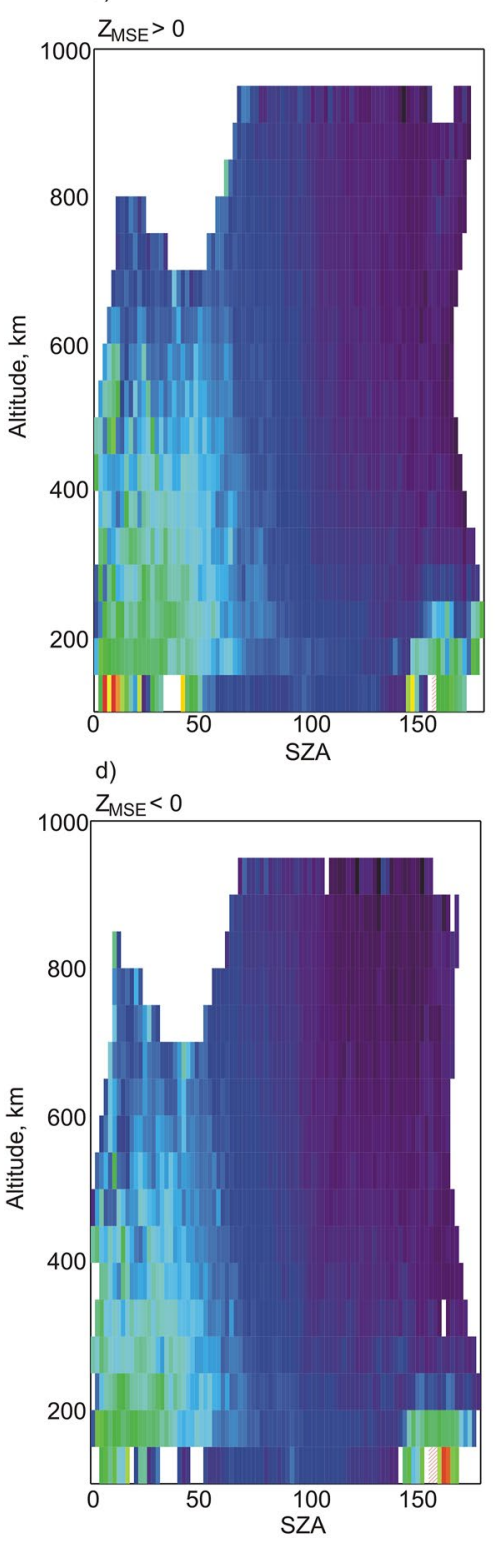

b)

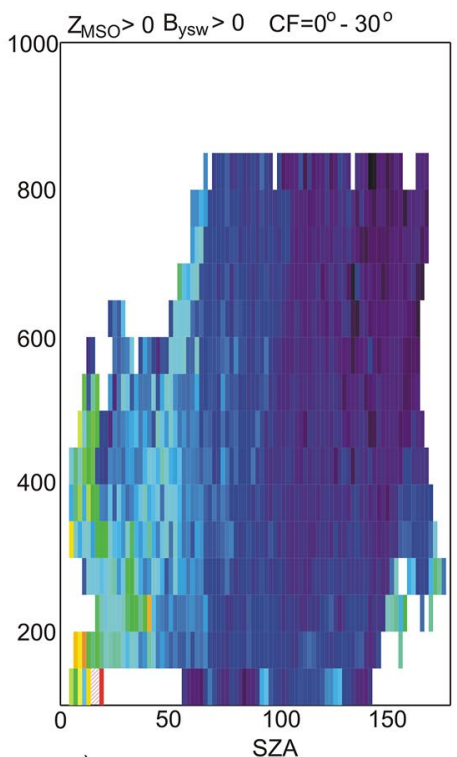

e)

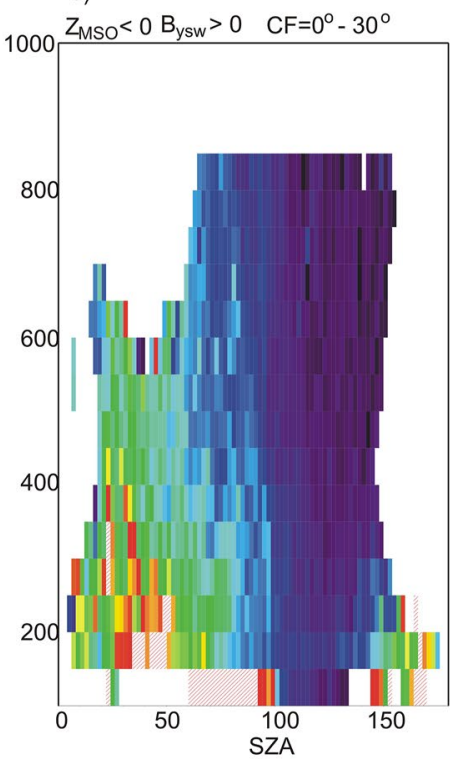

c)

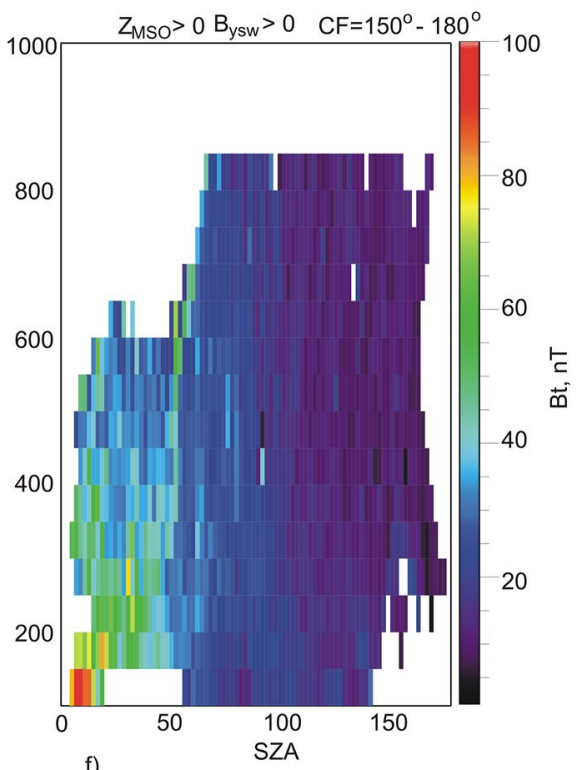

f)

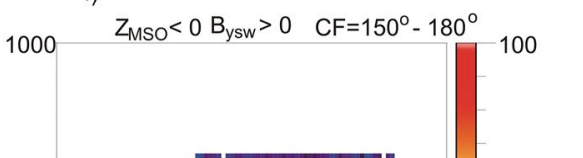

80

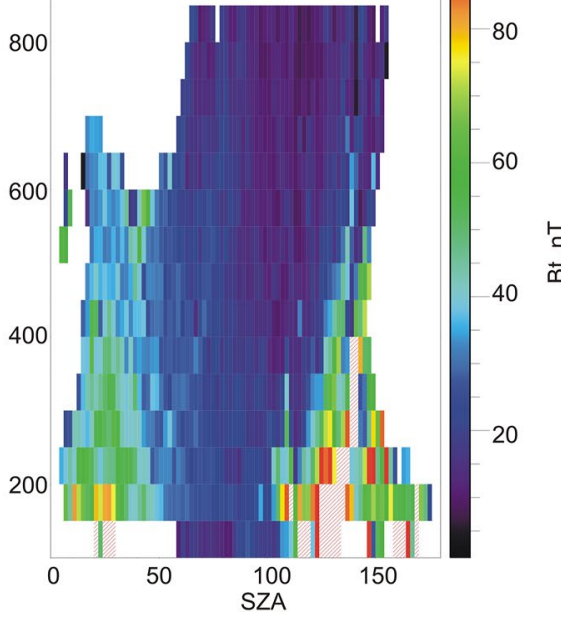

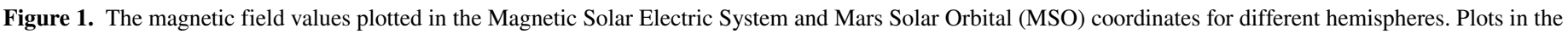

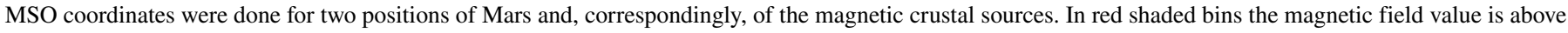
100 nT.

transmitted at the same time. We take a subset of these products (c0,cf,d1,ce,d0,cd,cc,ca - depending on availability) to reconstruct a matrix $M$ with the dimension of 32 energy bins, 4 mass bins, 16x4 field of view bins and $4 \mathrm{~s}$ time resolution by linear interpolation from this subset. In each interpolation step we normalize the respective dimension to guarantee that the sums over all dimensions of $\mathrm{M}$ stays the same as for the original products. By this procedure we get a time series with commensurate sampling in energy, field of view, and time for the 4 major ion groups $\left(\mathrm{H}^{+}, \mathrm{He}^{++}, \mathrm{O}^{+}, \mathrm{O}_{2}^{+}\right)$. From this time series we calculate density, velocity, and temperature for these groups with even time sampling. Since the measurements of the low-energy ions in the dense ionosphere and in the planetary wake are affected by the spacecraft (s/c) potential we made corrections using the values of the s/c potential presented by the STATIC team. Calculations of the corrected moments from three-dimensional distribution functions were based on Liouvilles theorem (see, for example, Lavraud \& Larson, 2016). Here we focus only on the observations of the flux of the oxygen ions $\left(\mathrm{O}^{+}\right.$and $\mathrm{O}_{2}^{+}$together) which dominate in the topside ionosphere and in the tail. The STATIC observations were complemented by the measurements of the magnetic 


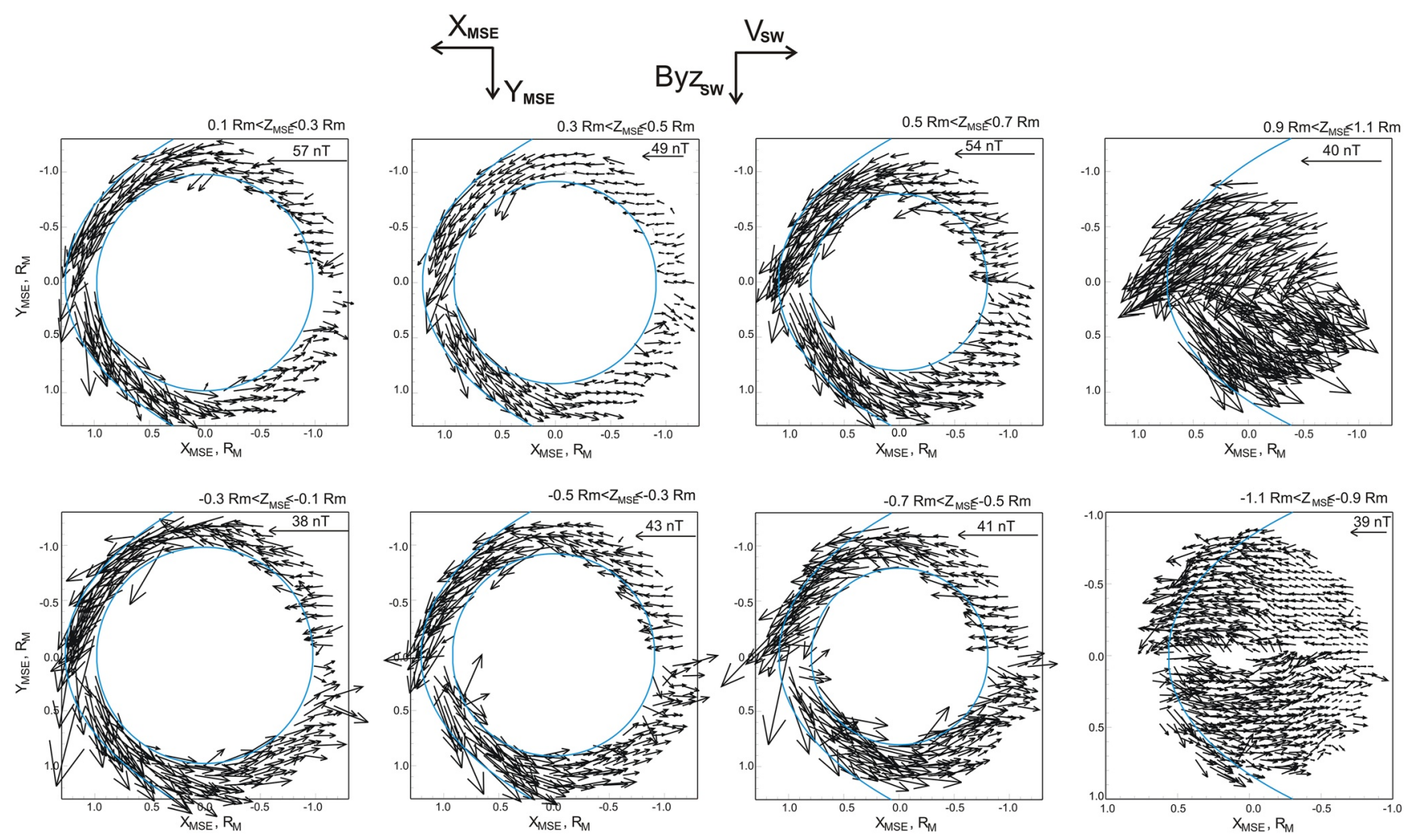

Figure 2. Projections of the magnetic field onto XY planes at different $Z_{\mathrm{MSE}}$ distances in $E^{+}$(upper row) and $E^{-}$(lower row) hemispheres. Blue curves show crosssections of Mars and the induced magnetosphere boundary (November 2014-August 2017).

field (Connerney et al., 2015). We derive the IMF orientation by taking the median value of the magnetic field vector components from $30 \mathrm{~min}$ around the time where the maximum proton velocity measured by the solar wind monitor SWIA (Halekas et al., 2015) was observed. We do not exclude orbits with a high variation of the field vector. We processed all orbits in order to study the global features without any assumptions about stationarity of the IMF orientation. During the orbits when MAVEN was not in the solar wind we use the magnetic field measurements in the magneto sheath. Note that for these cases the clock angle errors due to the IMF deformations may appear (Dong et al., 2019).

\section{Observations}

We constrained our analysis to the region inside the nominal boundary of the induced magnetosphere of Mars (Dubinin \& Fraenz, et al., 2006) and altitudes below 1,000 km in the regions where the boundary is above this altitude.

Figure 2 shows projections of the magnetic field onto XY planes at different $Z_{\mathrm{MSE}}$ distances in the $E^{+}\left(Z_{\mathrm{MSE}}>0\right)$ (upper row) and $E^{-}\left(Z_{\mathrm{MSE}}<0\right)$ (lower row) hemispheres based on the median components of the magnetic field. Blue curves present the Mars position and position of the nominal magnetospheric boundary at the corresponding $\mathrm{XY}$ planes. The size of each spatial bin is $300 \mathrm{~km}$. We observe that in both the $E^{+}$and $E^{-}$dayside hemispheres draping of the IMF propagates down to low altitudes. At the nightside of the of $E^{+}$hemisphere the field lines slightly converge to the wake. With increase of $Z_{\mathrm{MSE}}$ this convergence disappears and the field lines begin to diverge in accordance with a classical draping topology. The picture is different in $E^{-}$hemisphere. Wrapping of the field lines increases with distance from the central $\mathrm{XY}$ plane and at $Z_{\mathrm{MSE}} \sim-1 R_{M}$ we observe an almost closing of the field lines (see the panel at $Z_{\mathrm{MSE}}=-0.9-1.1 R_{M}$ in which we use a bin size $100 \mathrm{~km}$ for a better spatial resolution).

Figure 3 shows the median values of the $B_{y}$-component of the magnetic field in the $E^{+}\left(Z_{\mathrm{MSE}}>0\right)$ and $E^{-}$ $\left(Z_{\mathrm{MSE}}<0\right)$ hemispheres as a function of altitude and solar zenith angle. This means each bin shows the net value 

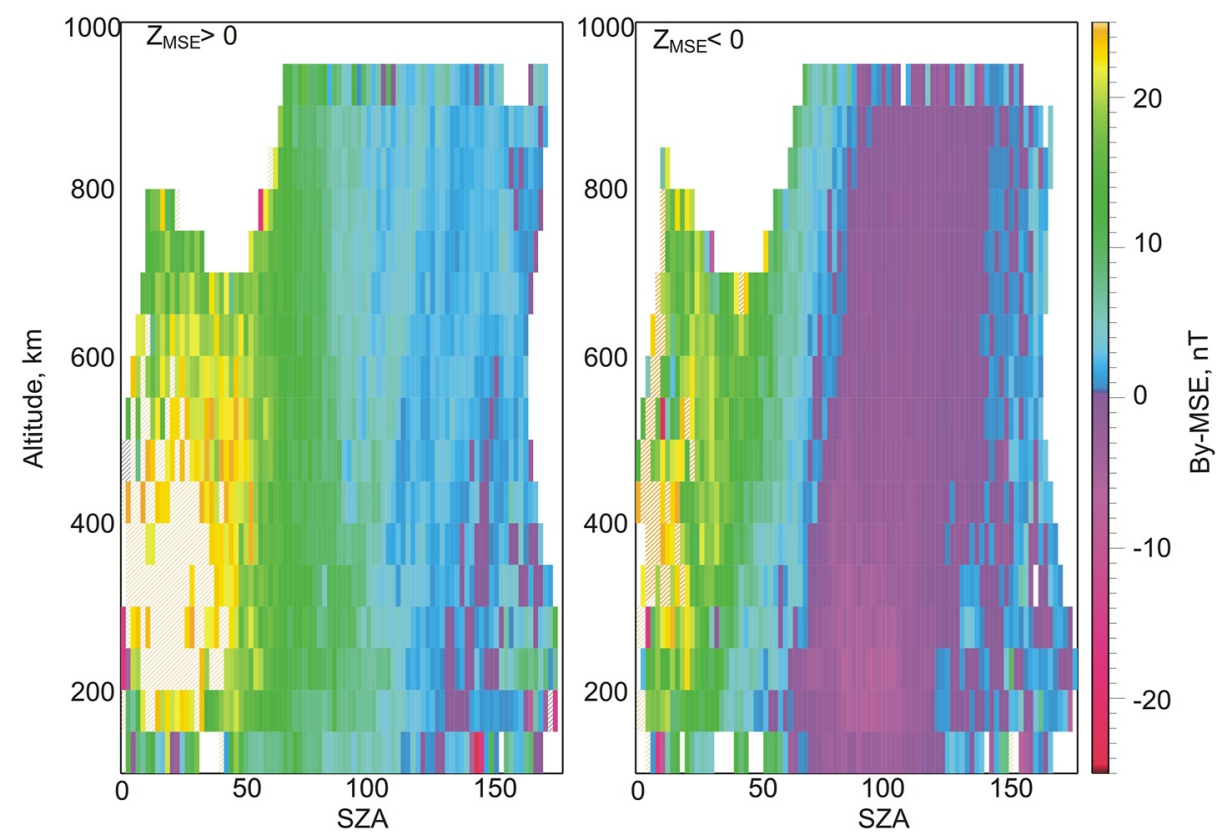

Figure 3. Median values of the $B_{y}$-component of the magnetic field in $E^{+}\left(Z_{\mathrm{MSE}}>0\right)$ and $E^{-}\left(Z_{\mathrm{MSE}}<0\right)$ hemispheres as a function of altitude and solar zenith angle (November 2014-May 2018) In red shaded bins the magnetic field value is above $30 \mathrm{nT}$.

of $B_{y}$ across a half-ring around the planet. A positive value then indicates that the induced field is aligned with $B_{\mathrm{IMF}}$, a negative values that it is opposite to $B_{\mathrm{IMF}}$. It is seen that a change of sign of the $B_{y}$ component in $E^{-}$hemisphere, that is a strong wrapping of the field lines around Mars already occurs at low altitudes. At $S Z A \geq 130^{\circ}$ $-150^{\circ}$ the field lines begin to diverge.

Figure 4 presents the projections of the median magnetic field unit vectors onto the MSE XY plane at $Z_{\mathrm{MSE}}=0.5-$ $0.7 R_{M}$ and $Z_{\mathrm{MSE}}=-0.5--0.7 R_{M}$ plotted in the variables altitude and SZA. That provides us a much better resolution $\left(50 \mathrm{~km}-2^{\circ}\right)$ than in Figure 2. It is observed that draping features propagate down to altitudes of 200-250 km.

Figure 5 (panels (a) and (b)) shows projections of the bulk velocity of oxygen ions onto the $X Y_{\text {MSE }}$ plane in the $E^{+}$and $E^{-}$hemispheres (bin size is $300 \mathrm{~km}$ ). Here we present the components of the bulk plasma velocity $V=\left(n_{O_{2}^{+}} V_{O_{2}^{+}}+n_{O^{+}} V_{O^{+}}\right) /\left(n_{O_{2}^{+}}+n_{O^{+}}\right)$. Although deviations exist, the ions generally exhibit a dayside to nightside flow.

Figure $5 \mathrm{c}$ shows projections of the bulk speed onto the XY plane at $Z_{\mathrm{MSE}}=0.9-1.1 R_{M}$, in which we observe a reversal of the velocity of the oxygen ions with the appearance of the sunward flow that indicates reconnection of the magnetic field in this area. This region is highlighted by the red vectors that also use a bin size of $100 \mathrm{~km}$.

Figure 6 depicts projections of the magnetic field onto the MSE XZ plane at different $Y_{\mathrm{MSE}}$ distances from the noon-midnight plane. The main features are the appearance of a large $B_{z}$ component and well ordered draping around Mars. At $-0.7 R_{M}<Y_{\mathrm{MSE}}<0.7 R_{M}$ draping persists even at the nightside. At $|Y|>0.7 R_{M}$ the geometry of a such draping becomes more and more asymmetrical. The vectors in the both $E^{+}$and $E^{-}$hemispheres show a systematic turn in $-\mathrm{Z}(+Z)$ directions at $+Y_{\mathrm{MSE}}\left(-Y_{\mathrm{MSE}}\right)$, respectively, and this indicates a draping of the field lines along the $Z$-axis with a regular counterclockwise rotation of the $B_{Y Z}$ vector in $E^{+}$hemisphere (Chai et al., 2019; Dubinin et al., 2019; Ramstad et al., 2020).

Figure 7 shows projections of the oxygen bulk velocity vectors onto the $X Z_{\mathrm{MSE}}$ - plane at different $Y_{\mathrm{MSE}}$ distances. The left panel presents the convolution of all measurements made at $Y_{\mathrm{MSE}}>0$. On the background of slow plasma motion from the dayside to the nightside we observe ion extraction and their notable acceleration mainly in the $+Z_{\mathrm{MSE}}$ direction. On the nightside, plasma tends to flow into the wake which is more evident in $E^{+}$hemisphere. 

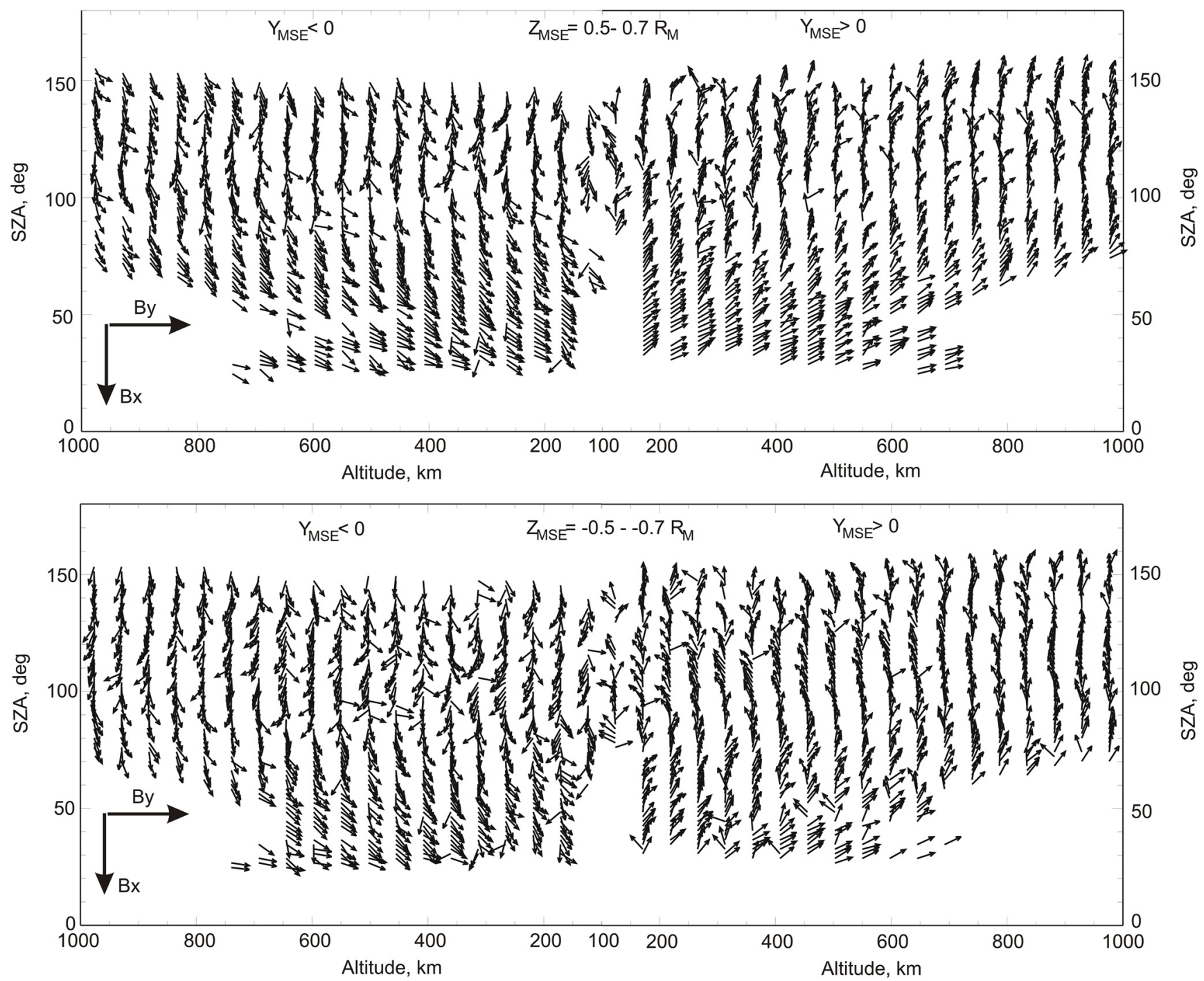

Figure 4. $B_{x y}$ projections of the unit vectors of the magnetic field at $Z_{\mathrm{MSE}}=0.5-0.7 R_{M}$ and $Z_{\mathrm{MSE}}=-0.5--0.7 R_{M}$ plotted in the variables altitude and SZA (November 2014-August 2017).
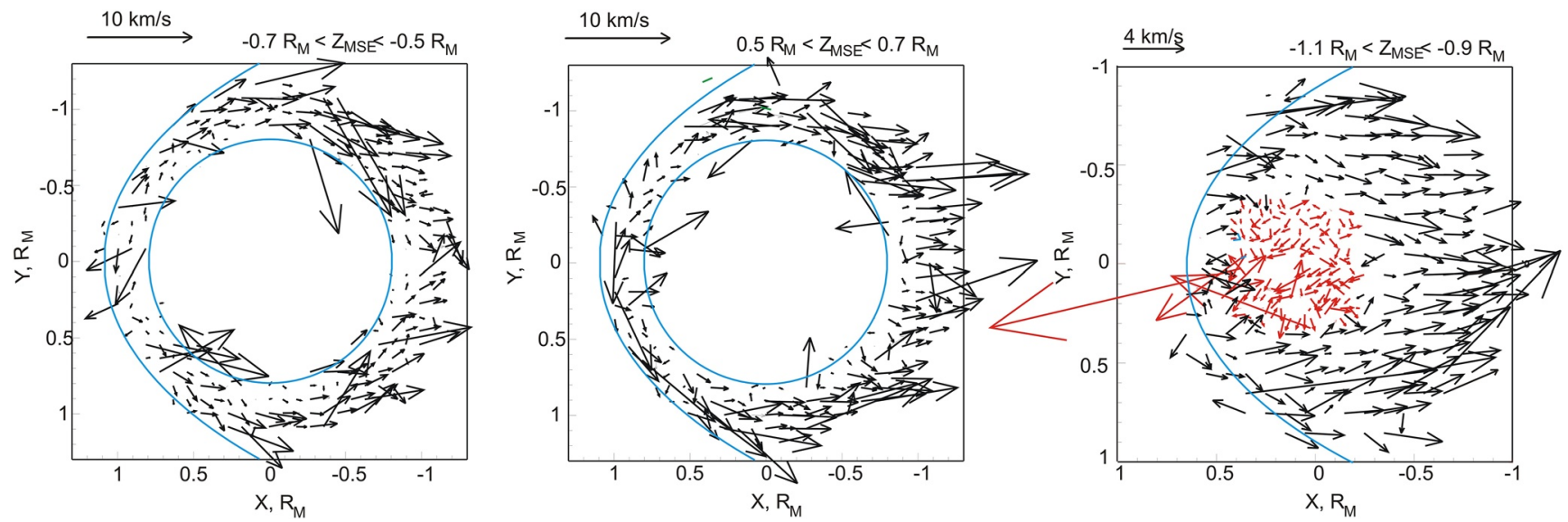

Figure 5. Projections of the bulk speed of oxygen ions onto two $X Y_{\mathrm{MSE}}$ planes in $E^{+}$and $E^{-}$hemispheres. In the area with red colored vectors we use a bin size of $100 \mathrm{~km}$ (November 2014-August 2017). 


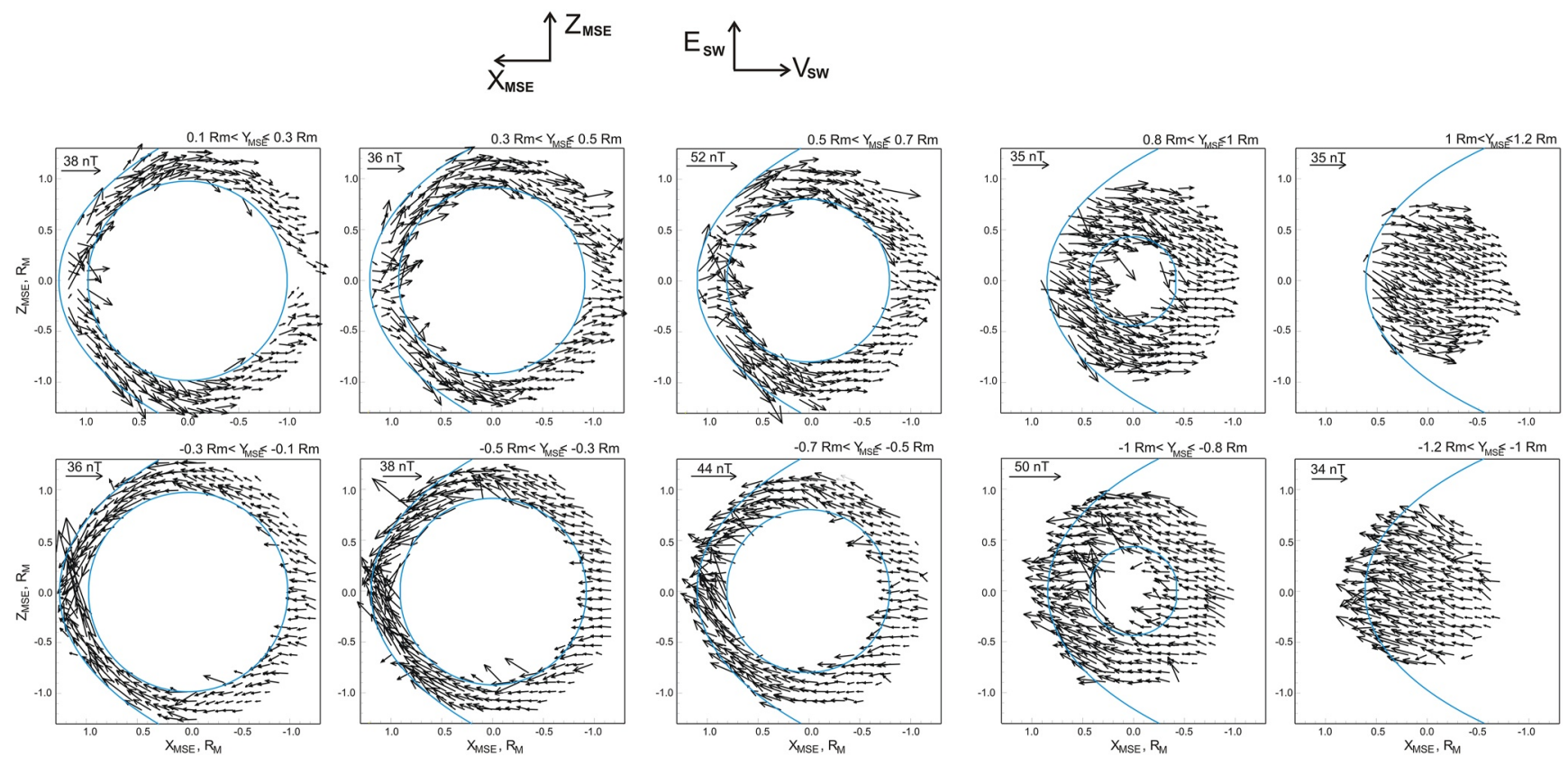

Figure 6. Projections of the magnetic field onto XZ planes at different $Y_{\mathrm{MSE}}$ distances from the noon-midnight plane (November 2014-August 2017).

To make this plot more compact some long vectors are green colored with the tagged values of the ion speed. They probably correspond to oxygen ions originated from the hot oxygen corona and accelerated outside the magnetosphere.
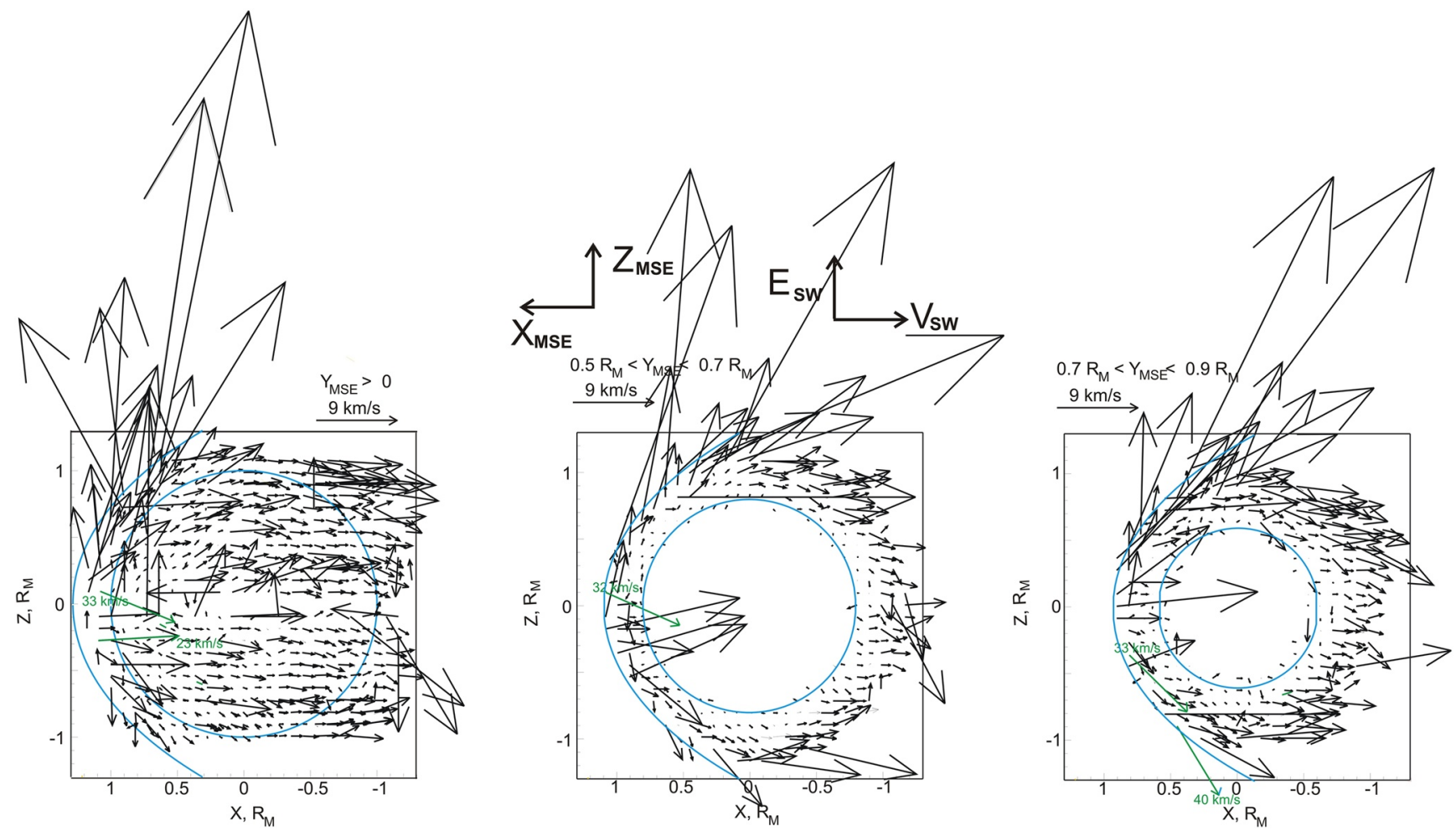

Figure 7. Projections of the oxygen bulk velocity vectors onto the $X Z_{\mathrm{MSE}}$ - planes (November 2014-August 2017). Green colored vectors with the tagged values correspond to large speeds of ions accelerated in the solar wind/magnetosheath. 

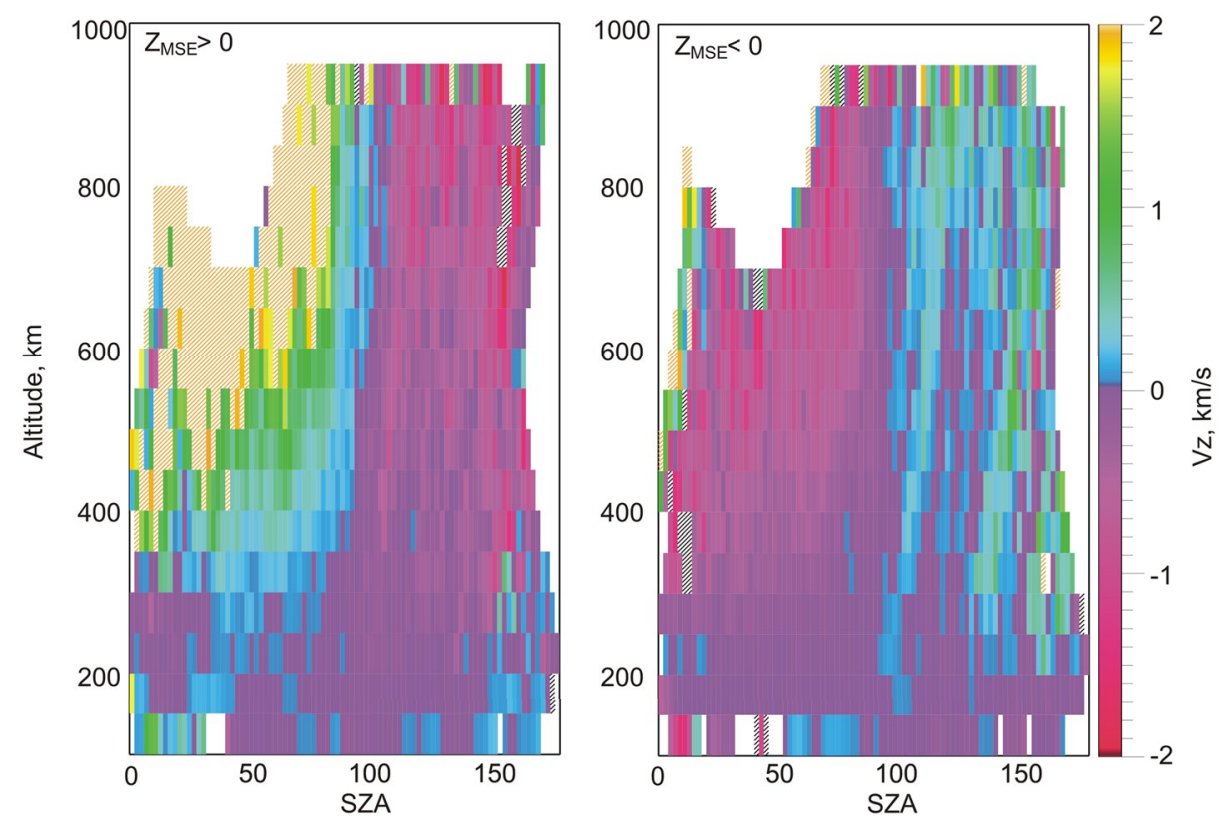

Figure 8. Median values of the $V_{Z}$ component in $E^{+}$and $E^{-}$hemispheres as a function of altitude and solar zenith angle. In shaded by red (black) bins $V_{z}>2\left(V_{z}<-2\right) \mathrm{km} / \mathrm{s}$ (November 2014-August 2017).

Figure 8 shows the median $V_{Z}$ velocity component in $E^{+}$and $E^{-}$hemispheres as a function of altitude and solar zenith angle. As in Figure 3 each bin shows the median of the quantity across a half-ring around the planet. To see where acceleration of oxygen ions starts the color bar is oversaturated. Velocities in red and black dashed bins are higher (lower) than $+/-2 \mathrm{~km} / \mathrm{s}$, respectively. At the dayside of the $E^{+}$hemisphere $\left(\mathrm{SZA}<\sim 90^{\circ}\right)$ ions are moving in $+Z_{\mathrm{MSE}}$ direction as one may expect for plasma fluid flowing around Mars. The velocity generally increases with altitude. It is observed that beginning of a regular ion acceleration in $E^{+}$hemisphere occurs at $\sim 300$ $\mathrm{km}$. On the nightside $\left(\mathrm{SZA}>90^{\circ}\right.$ ) plasma flows in the opposite direction supplying the nightside ionosphere. In the $E^{-}$hemisphere ion velocities are smaller. At the dayside $\left(\mathrm{SZA}<90^{\circ}\right)$ the oxygen ions are moving in the $-Z_{\mathrm{MSE}}$ direction following the flow of the shocked solar wind in the adjacent magnetosheath. At the night side the ionospheric plasma flows upwards $\left(V_{z}>0\right)$ also filling the nightside ionosphere.

Extraction of ions from the ionosphere with their following acceleration at the dayside might be related with draping features of the magnetic field lines. Figure 9a shows the $B_{Y Z}$ projections of the magnetic field measured at the dayside onto the $Y Z_{\mathrm{MSE}}$ plane looking at the planet from the solar wind perspective. In both $E^{+}$and $E^{-}$hemispheres, we observe draping features with a different sign of the field bending indicating that the central parts of the field lines are moving slower than their rear parts. That implies the initiation of the magnetic tail formation already at the dayside. A geometry of the field bending occurs different in both hemispheres. In the $E^{+}$hemisphere, the $B_{Z}$ component becomes significant only in the central part, near the noon-midnight plane, while in the $E^{-}$hemisphere the region with a large $B_{Z}$ is much broader although the tension forces are also stronger near the noon meridian enhancing the plasma drag force in the ionosphere in the $+/-Z_{\mathrm{MSE}}$ directions.

Figure $9 \mathrm{~b}$ depicts the $V_{Y Z}$ projections of the oxygen bulk speed. In the $E^{+}$hemisphere, the ions gain more velocity, producing ion beams with energies that increase with altitude (Dubinin, Fraenz, et al., 2006; Dubinin, Ludin, et al., 2006). This enhancement of $V_{z}$ in the $E^{+}$-hemisphere is clearly shown in Figure 9c which depicts the projections of the ion speeds measured by STATIC at $0<X<1 R_{M}$ onto the whole $Y Z_{\mathrm{MSE}}$ plane sampled by MAVEN. The observed fan of extracted and accelerated oxygen ions corresponds to a pickup ion population originated already at high altitudes of the upper ionosphere.

A strong asymmetry in the motion of oxygen ions is also shown in Figures $9 \mathrm{~d}$ and $9 \mathrm{e}$ which depict the $V_{z}$ and $V_{y}$ components of the velocities measured at $0<X<1 R_{M}$. White dotted circle in these panels represent the nominal position of the magnetosphere boundary in the terminator plane. The region inside this circle corresponds to the ionospheric area discussed in the paper. Here plasma moves parallel to the motion of the solar wind in the 


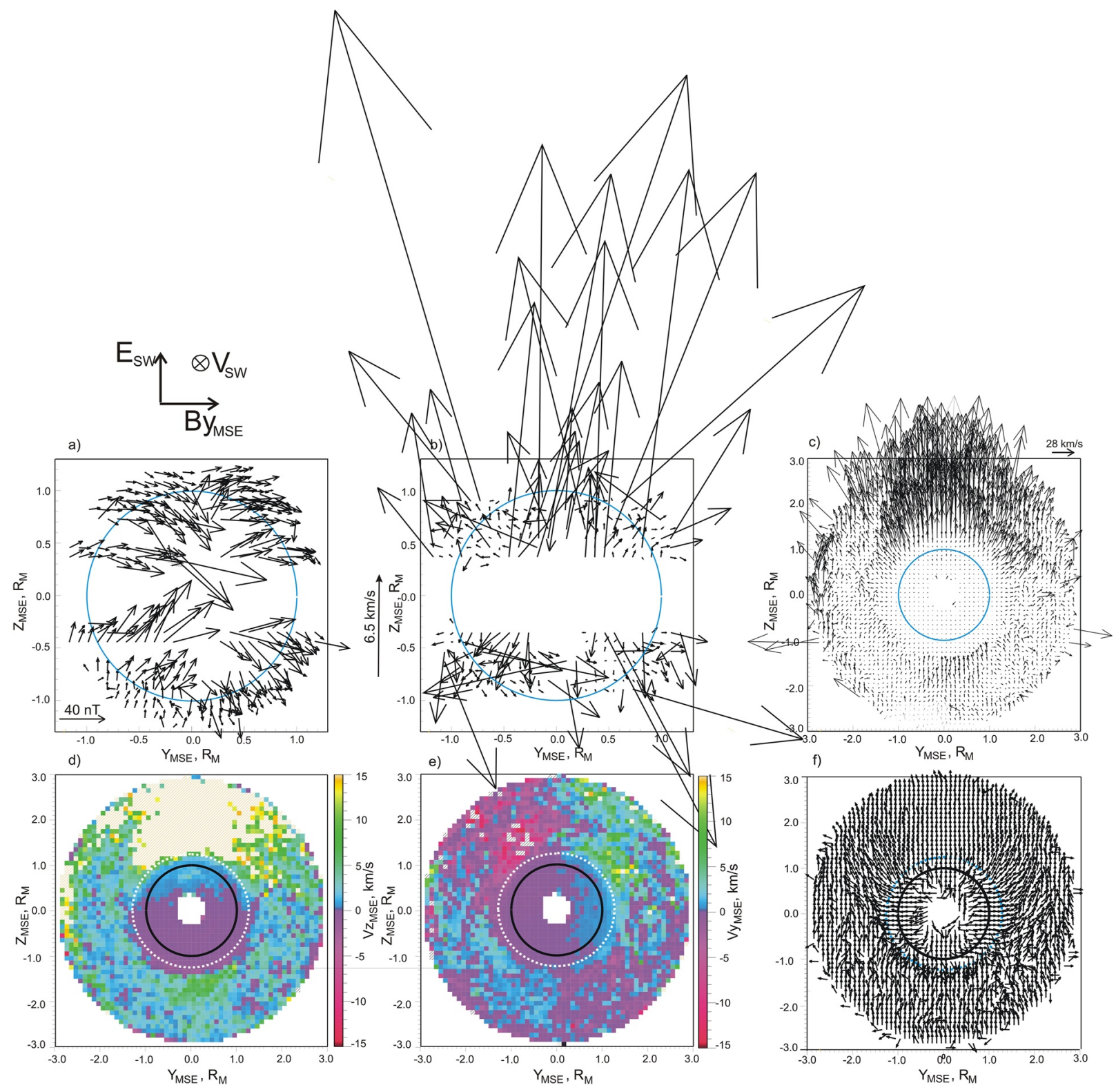

Figure 9. (a) $B_{Y Z}$ projections of the magnetic field onto the $Y Z_{\mathrm{MSE}}$ plane measured at the dayside at $Z$-cross sections at altitudes below $1,000 \mathrm{~km}$ and within the nominal induced magnetosphere boundary. (b) $V_{Y Z}$ projections of the oxygen bulk speed. (c) $V_{Y Z}$ projections of the oxygen bulk speed for the whole $Y Z-M S E$ plane sampled by MAVEN at $0<X<1 R_{M}$ (d, e) Median components $V_{z}$ and $V_{y}$ of the ion velocity measured at $0<X<1 R_{M}$. In shaded red bins $V_{z}>15 \mathrm{~km} / \mathrm{s}$. The dotted white circle is the nominal position of the induced magnetosphere boundary in the terminator plane. (f) The projections of unit vectors of the oxygen ion flows onto the YZ-plane (November 2014-August 2017).

magnetosheath symmetrically flowing around Mars. Such motion across the draping magnetic field induce the electric fields $-V \times B$, but with much smaller values than in the adjacent magnetosheath. Ions freshly generated by photoionization will either be quickly assimilated into this flow or - if they are close to the boundary and gain a sufficient speed - can escape and be picked up by the much higher motional electric field in the sheath. Outside of the dotted circle the motion of the oxygen ions is governed by the direction of motional electric field in the magnetosheath. In $E^{+}$hemisphere, $V_{z}>0, V_{y}>0$ for $Y>0$ and $V_{y}<0$ for $Y<0$, while in $E^{-}$hemisphere, $V_{z}>0$, $V_{y}<0$ for $Y>0$ and $V_{y}>0$ for $Y<0$ (Figures $9 \mathrm{~d}$ and 9e). The ion motion in both hemispheres is depicted by Figure $9 \mathrm{f}$ which shows the projections of the unit vectors of the oxygen $\left(\mathrm{O}^{+}\right.$and $\left.\mathrm{O}_{2}^{+}\right)$ion flows onto the YZ-plane. Oxygen ions originated in the solar wind and sheath seem to flow around the magnetospheric obstacle. Ions of the ionospheric origin contribute to this flow at flanks and in polar region of the $E^{+}$hemisphere. Near the pole 


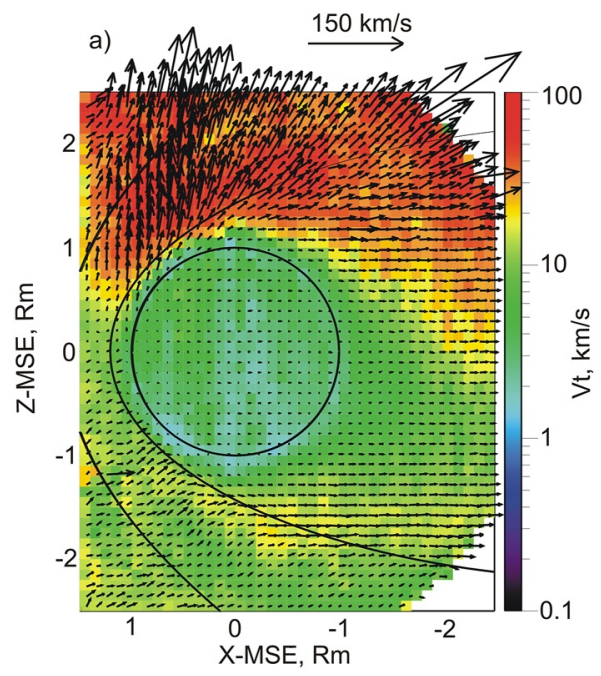

b)

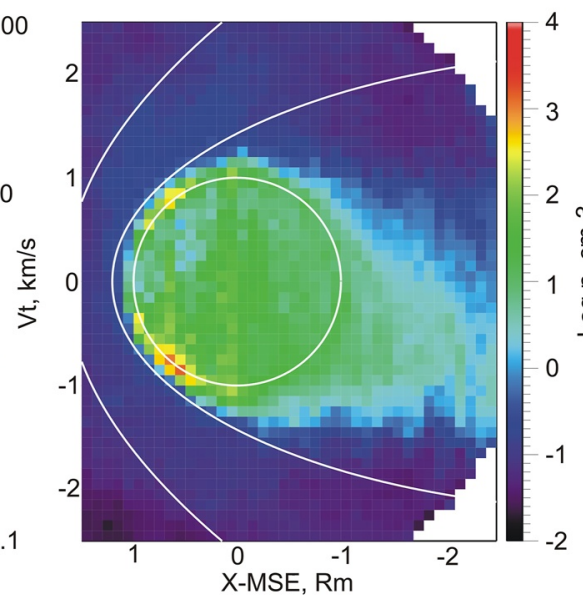

c)

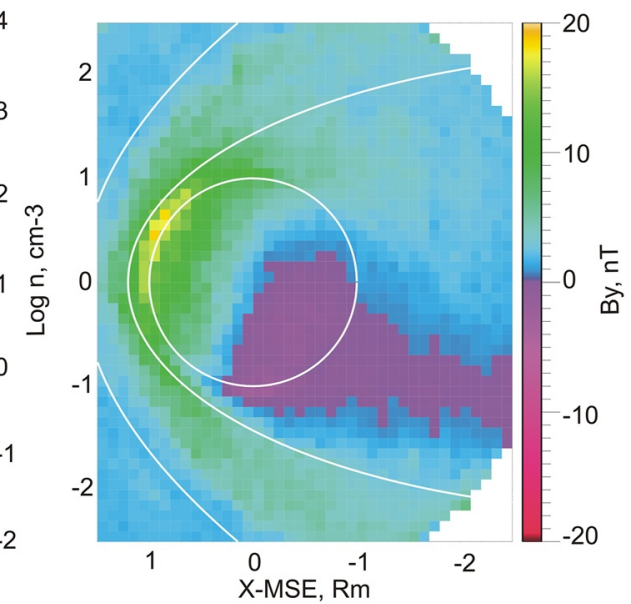

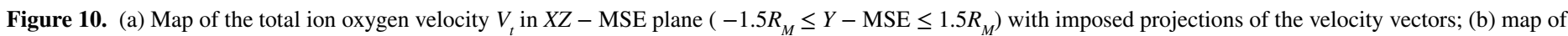
the ion oxygen density; (c) map of the $B_{y}$ component of the magnetic field (November 2014-May 2018).

region of the $E^{-}$hemisphere, flows within and outside the magnetosphere meet together and turn tailward. This flow is further shown in Figure 10a which shows a map of the projections of the velocity vectors of oxygen ions in the $X Z-M S E$-plane $\left(-1.5 R_{M} \leq Y-M S E \leq 1.5 R_{M}\right)$ imposed onto a map of the total value of the velocity. The observed asymmetry in ion flows in $E^{+}$and $E^{-}$hemispheres leads to the asymmetry in the distribution of the ion density in the tail and formation of the ion trail (Figure 10b). In the trail plasma is denser and moves tailward with slower speed because the magnetic tension forces begin to act in the sunward direction $\left(B_{y}\right.$-component of the magnetic field changes sign (Figure 10c).

To examine the role of the magnetic tension forces in the ion acceleration at the dayside we plot the absolute value of the $V_{z}$ component of the bulk speed as a function of $B_{x}$ at different altitudes in the $E^{+}$and $E^{-}$hemispheres (Figure 11, upper row). A change of sign of the $B_{x}$ component corresponds to crossing of the emerging tail current sheet. We observe two different regions with noticeable ion acceleration. One region is near the current sheet where the tension forces are stronger, and another one at large $\left|B_{x}\right|$ that matches the region adjacent to the induced magnetosphere boundary with pileup of the IMF. We also observe that ion acceleration by tension forces is more efficient in the $E^{+}$hemisphere. Similar features are seen while plotting the horizontal component of the ion velocity (Figure 11, lower row).

A difference in the magnetic field bending in the two hemispheres is well seen in Figure 12 which shows the $B_{y z}$ projections of the unit vectors of the magnetic field at $Z_{\mathrm{MSE}}=0.5-0.7 R_{M}$ and $Z_{\mathrm{MSE}}=-0.5--0.7 R_{M}$ plotted as a function of the variables altitude and SZA. At the dayside, at small SZA of the $E^{+}$hemisphere bending of the field lines is strong at all altitudes. With increase of SZA the bending becomes at first weaker while at $S Z A>$ $\sim 70^{\circ}$ and at the nightside the $B_{z}$ component changes sign and prevails. At the dayside of the $E^{-}$hemisphere both components are comparable at all altitudes implying a broader expansion of the bending features that might lead to a bulk plasma motion in the $-Z_{\mathrm{MSE}}$ direction. At the nightside the horizontal $B_{y}$ component dominates but the picture becomes much less regular with a frequent reversal of the sign of the $B_{y}$ component.

\section{Discussion}

The Martian magnetosphere contains elements of induced and intrinsic origin (see e.g., Chai et al., 2019; DiBraccio et al., 2018; Dubinin et al., 2017; Ramstad et al., 2020; Weber et al., 2019; Xu et al., 2017, 2018, 2020). To display these components one must use specific coordinate systems. Because of a strong asymmetry of the magnetosphere controlled by the direction of the motional electric field, the MSE coordinate system adequately describes aspects of the induced magnetosphere but loses features caused by the presence of the local crustal magnetizations. To study effects of the induced origin we used here the MAVEN data obtained for the period from November 2014 to May 2018 and utilized the MSE coordinate system. One should keep in mind that the 

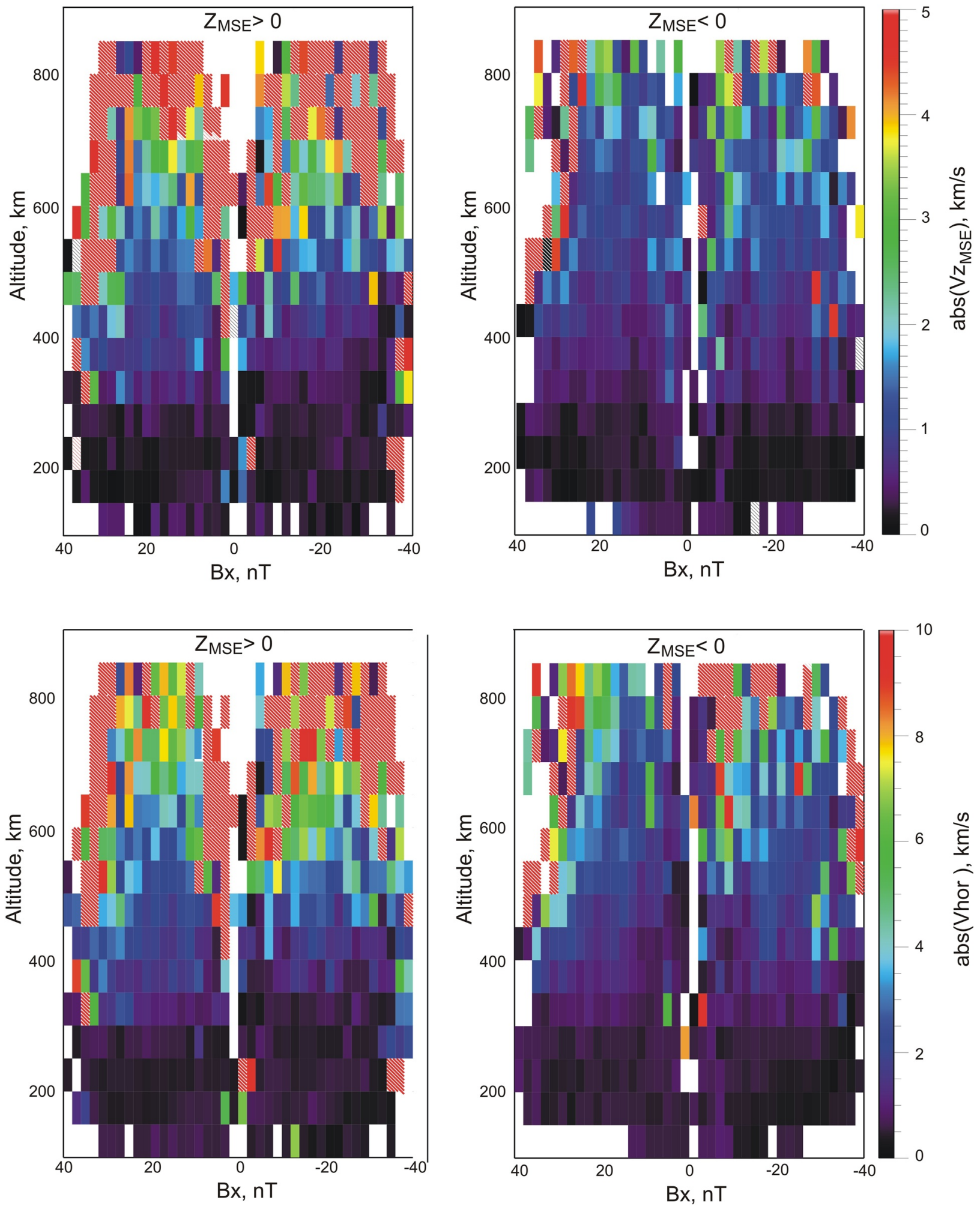

Figure 11. $V_{z}$-component of the velocity measured at the dayside as a function of $B_{x}$ component of the magnetic field and altitude. In shaded by red bins the velocity is higher than $5 \mathrm{~km} / \mathrm{s}$ (November 2014-August 2017). 

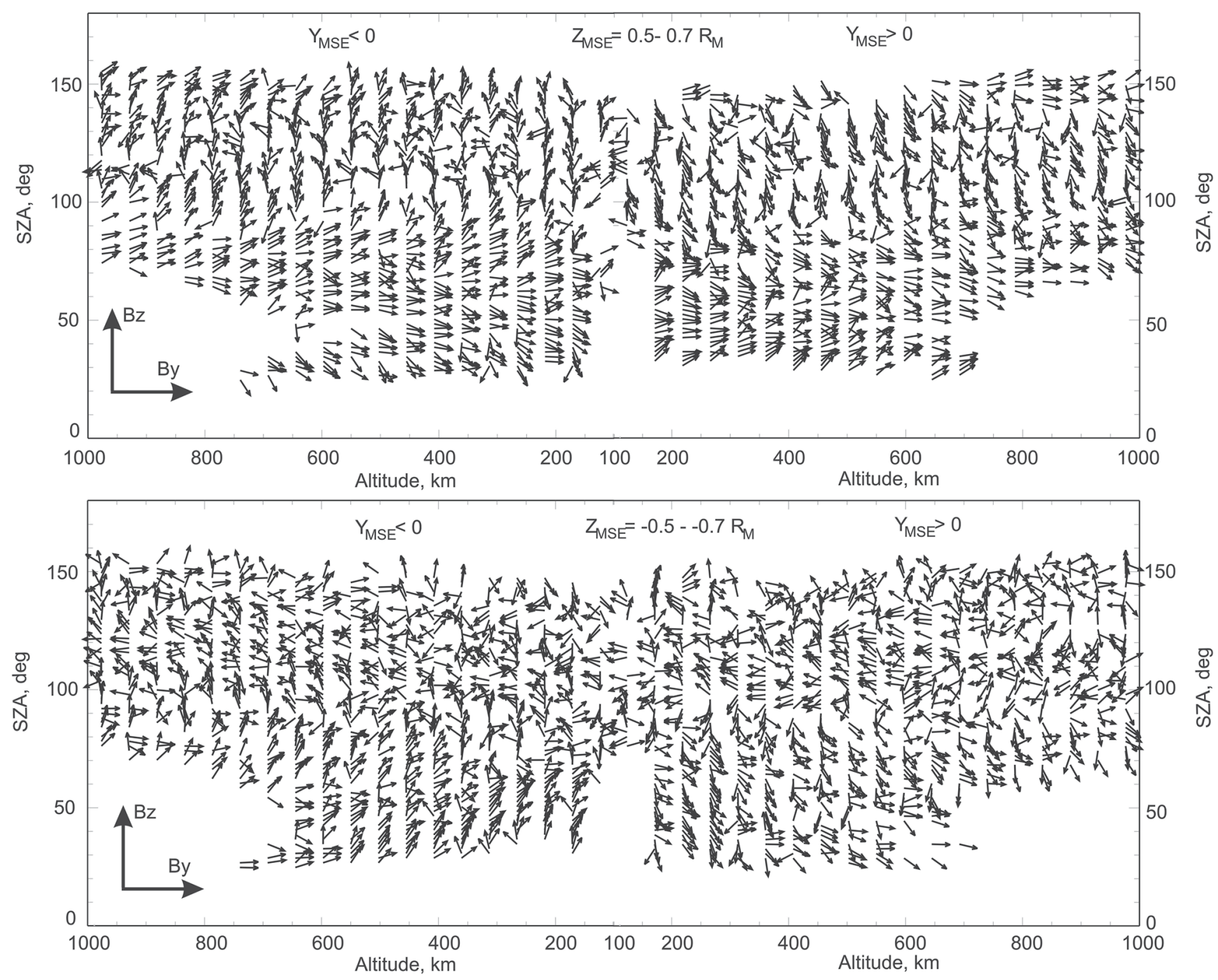

Figure 12. $B_{y z}$ projections of the unit vectors of the magnetic field at $Z_{\mathrm{MSE}}=0.5-0.7 R_{M}$ and $Z_{\mathrm{MSE}}=-0.5--0.7 R_{M}$ plotted in the variables altitude and SZA (November 2014-May 2018).

presented distributions of the magnetic field and plasma motions are not snapshots of the different cross-sections of the Martian magnetosphere, but rather characterize only the main properties of the induced magnetosphere but removes/suppresses features caused by the crustal magnetic sources while rotating the spacecraft orbits to the MSE-coordinate system and averaging over many orbits. The solar winds interactions with magnetized and nonmagnetized planets share many similarities in their features upstream of the bow shock, in processes at the bow shock itself, and in the magnetosheath (J. S. Halekas et al., 2017; Mazelle et al., 2004). However, the structures of the inner magnetosphere is very different. Although solar wind is mostly terminated at a certain distance from Mars by the induced magnetic field barrier formed by the draping of the IMF lines, the draping IMF penetrates deep into the ionosphere because the solar wind dynamic pressure generally exceeds the thermal plasma pressure in the upper Martian ionosphere. We show that draping features propagate down to low altitudes $(\sim 200-250 \mathrm{~km})$ sampled by MAVEN and envelop the planet.

When looking at the draping of the IMF, which penetrates into the ionosphere, in planes parallel to the central $X Y$ plane, which contains the IMF, we observe that draping differs between the $E^{+}$and $E^{-}$hemispheres. A wrapping of the field lines becomes stronger and stronger when approaching the $E^{-}$'polar region' in the MSE-coordinates and then reconnection signatures with reversal of ion flows appear. Note that the values of the sunward ion speeds are significantly less than the Alfven speed implying only a partial ion coupling (see also Harada et al., 2020). The hybrid simulations of the solar wind/Mars interaction (Dubinin et al., 2019) confirm the permanent existence of a 
such area. Observations by Harada et al. (2017) of the trapped electrons with two-sided loss cones near the terminator, in the area not connected to the strong crustal sources, also support the reconnection scenario in this region. Mechanisms responsible for such a permanent reconnection in this area still remain unknown. This phenomenon is probably related to the formation of the plasma bulge in this region due to convergence of the ion fluxes in the $E^{-}$hemisphere and a reduced ion outflow due to a wrapped field geometry (see Figure 10). In hybrid simulations (Dubinin et al., 2019) performed for fixed upstream conditions with $B_{\text {ysw }}>0$ and without the crustal fields, such bulge appears in the region near $X \sim 0$ and $Z_{\mathrm{MSE}} \sim-1 R_{M}$ and then spreads to the tail. The observations show a broader region with the increased plasma density and a general shift of the ionosphere in the $-Z_{\mathrm{MSE}}$ direction ion trail. It is assumed that such a shift is a result of the recoil effect due to ion extraction in the $+Z_{\mathrm{MSE}}$ direction although the recoil forces are not well identified. Dubinin et al. (2019) suggested that these forces are caused by the arising additional magnetic field draping along the $Z$-axis (see also Chai et al., 2019; Ramstad et al., 2020).

Asymmetrical wrapping of the magnetic field lines was also observed in the near Venus tail (Zhang et al., 2010) implying that this feature might be typical for the induced magnetospheres.

We also see a slipping of the ionospheric plasma around Mars from the dayside to the nightside with a gradual ion acceleration. Near the terminator the bulk velocity $V=\left(n_{O_{2}^{+}} V_{O_{2}^{+}}+n_{O^{+}} V_{O^{+}}\right) /\left(n_{O_{2}^{+}}+n_{O^{+}}\right)$achieves $\sim 3-4 \mathrm{~km} / \mathrm{s}$. At the nightside the velocity reaches $\sim 10 \mathrm{~km} / \mathrm{s}$. This motion might be driven either by the thermal pressure gradient (Ergun et al., 2016) or/and by the magnetic tension forces. According to Collinson et al. (2019) and Xu et al. (2018) the potential drop of the ambipolar electric field near the terminator is $\leq 1.5 \mathrm{~V}$ that corresponds to $\sim 3$ and $\sim 4.4 \mathrm{~km} / \mathrm{s}$ for $O_{2}^{+}$and $O^{+}$ions, respectively, that could explain the observed ion speeds. Further, at the nightside, contribution of $j \times B$ forces probably dominates.

Projections of the magnetic field onto the $X Z$-planes reveal another interesting feature of the field topology - the appearance of a large $B_{z}$ component. At the dayside the central parts of the field lines, immersed in a denser ionosphere, are moving slower than their rear parts. As a result, a bending of the field lines with different $\operatorname{sign}$ in $E^{+}$ and $E^{-}$hemispheres arises. Such bending gives start to formation of the current sheet of the magnetotail (see also J. S. Halekas et al., 2006). Bending of the field lines propagates to low altitudes $(\sim 200 \mathrm{~km})$ and its geometry occurs different in both hemispheres. In the $E^{+}$hemisphere the bending is more localized around the noon-midnight plane while in the $E^{-}$hemisphere bending spreads to a much broader area. With distance from the noon-midnight plane $\left(|Y|>0.7 R_{M}\right)$ the field topology becomes more and more asymmetrical between the $E^{+}$and $E^{-}$hemispheres with a turn of the magnetic field lines into the $-Z_{\mathrm{MSE}}$ direction. Such a twist gives rise to a peculiar draping along the $Z$-axis (Chai et al., 2019; Dubinin et al., 2019; Ramstad et al., 2020).

The asymmetry is also evident in plasma flows. Although the ionospheric plasma flow in both hemispheres is parallel to the shocked solar wind in the magnetosheath (a comet-like fashion of the flow, Lundin et al., 2008) the velocities in the $E^{+}$hemisphere are higher implying the important role of the direction of the motional electric field in the solar wind. Acceleration of ions starts at rather low altitudes $(\sim 250-300 \mathrm{~km})$ and continues with gain in ion energy at higher altitudes. These ions give origin to ion beams and ion plume observed at higher altitudes in the $E^{+}$hemisphere (Dong et al., 2015; Dubinin, Fraenz, et al., 2006; Dubinin, Ludin, et al., 2006). We observe that the magnetic field tensions related to the strong bending of the field lines in the $E^{+}$hemisphere can accelerate ions from $\sim 300 \mathrm{~km}$ and lift them to altitudes at which the motional electric field further accelerates them to much higher speeds. With increase of altitude the asymmetry of oxygen ion motions between $E^{+}$and $E^{-}$hemispheres becomes more and more significant and in the adjacent magnetosheath is determined by the direction of the motional electric field driven by the shocked solar wind - in the $E^{+}\left(E^{-}\right)$hemisphere, ions are moving outward (inward) to the planet, respectively.

In conclusion, Figure 13 shows a sketch illustrating the main features of the asymmetry in the magnetic field topology and plasma flow within the induced magnetosphere of Mars. Panels (a) and (b) depict views from Sun and from the dusk side, respectively. We show that the IMF penetrates very deep to the ionosphere draping around the planet. Plasma in the upper ionosphere $(h \geq \sim 250-300 \mathrm{~km})$ is not at rest, but is driven into motion from the dayside to the nightside and further to the tail, streaming parallel to the shocked solar wind in the adjacent magnetosheath but with much smaller velocities. The motion is driven by the gradients of the thermal and the magnetic pressure, and by the magnetic field tensions. As a result, such a motion is not axially symmetrical. An asymmetry between $E^{+}$and $E^{-}$hemispheres controlled by the direction of the motional electric field in the solar wind also exists implying penetration of the attenuated motional electric field into the ionosphere. Extraction of 


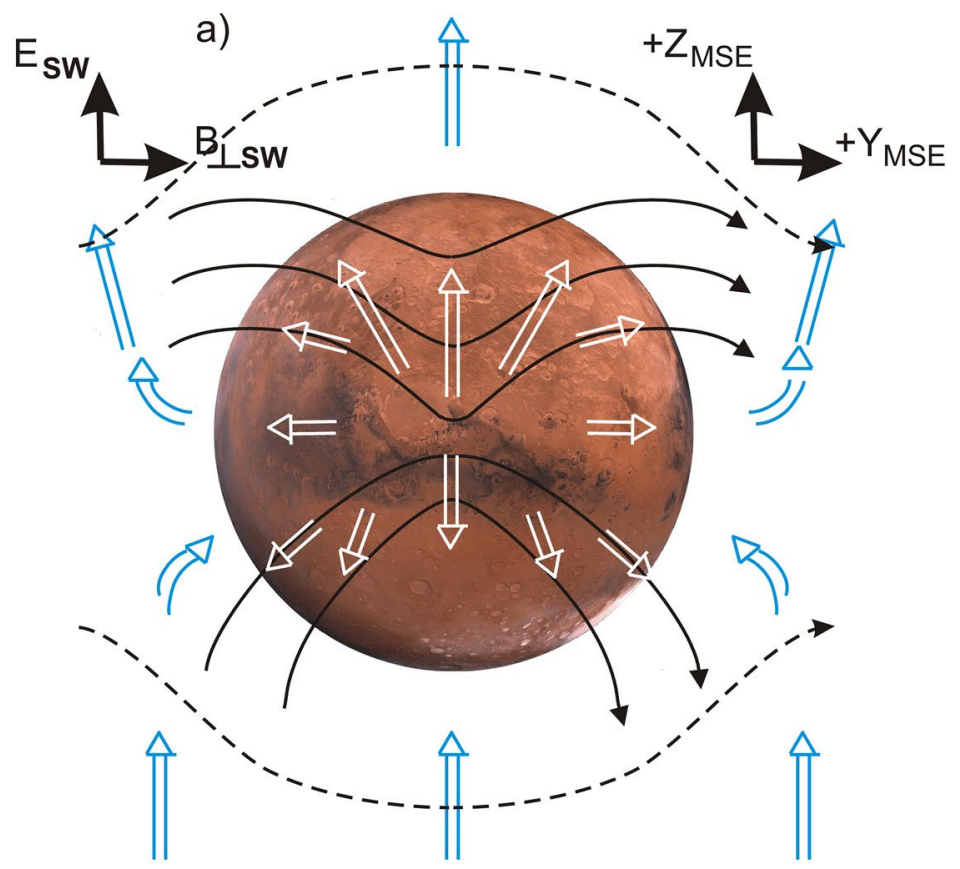

b)

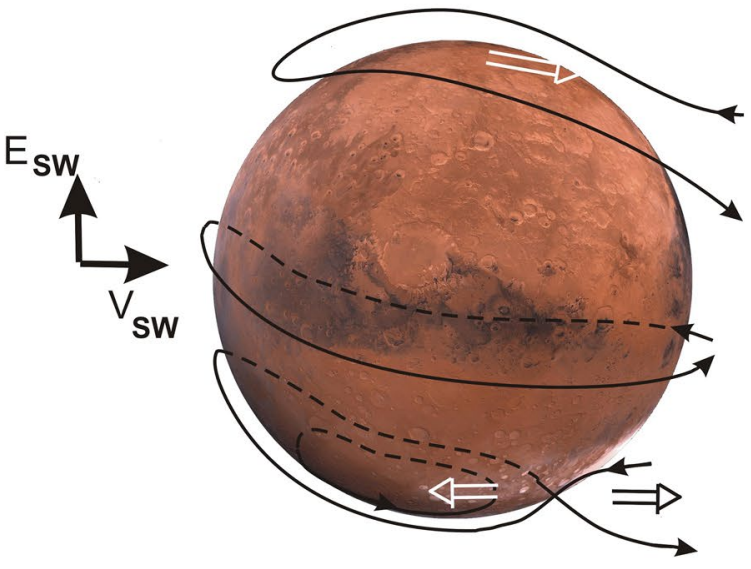

Figure 13. (a) Sketch of the field topology and plasma motions at the dayside magnetosphere in the $Y Z$ plane. Solid black lines are the magnetic field lines within the induced magnetosphere. Dashed lines are field lines in the sheath. Open white arrows depict the motion of the oxygen ions within the magnetosphere. Open blue arrows show the flow of oxygen ions originated from the hot oxygen corona outside the magnetosphere. (b) View from $+Y_{\mathrm{MSE}}$ axis.

Acknowledgments

The Mars Atmosphere and Volatile Evolution project is supported by NASA through the Mars Exploration Program. Authors E. Dubinin, M. Pätzold, S Tellmann. Wish to acknowledge support from DFG for supporting this work by grants TE 664/4-1 and PA 525/25-1. O. Vaisberg, S. Shuvalov and L. Zelenyi wish to acknowledge support from the Russian Science Foundation by grant 21-42-04404. ions and their significant acceleration in the $E^{+}$ionosphere is accompanied by a recoil effect of the bulk ionosphere in the opposite direction that produces a strong wrapping of the magnetic field lines around Mars and their subsequent reconnection.

\section{Data Availability Statement}

Mars Atmosphere and Volatile Evolution data are publicly available through the Planetary Data System (https:// pds-ppi.igpp.ucla.edu/mission/MAVEN).

\section{References}

Acuña, M. H., Connerney, J. E. P., Ness, N. F., Lin, R. O., Mitchell, D., Carlson, C. W., et al. (1999). Global distribution of crustal magnetism discovered by Mars Global Surveyor MAG/ER experiment. Science, 284, 790-793. https://doi.org/10.1126/science.284.5415.790

Chai, L., Wan, W., Wei, Y., Zhang, T., Exner, W., Fraenz, M., et al. (2019). The induced global looping magnetic field on Mars. The Astrophysical Journal Letters, 871(2), L27. https://doi.org/10.3847/2041-8213/aaff6e

Collinson, G., Gloser, A., Xu, S., Mitchell, D. L., Frahm, R., Grebowsky, L., et al. (2019). Ionospheric ambipolar electric fields of Mars and Venus. Comparisons between theoretical predictions and direct observations of the electric potential drop. Geophysical Research Letters, 46, 1168-1176. https://doi.org/10.1029/2018GL080597

Connerney, J. E. P., Espley, J., Lawton, P., Murphy, S., Odom, J., Olivers, R., \& Sheppard, D. (2015). The MAVEN magnetic field investigation. Space Science Reviews, 195(1-4), 257-291. https://doi.org/10.1007/s11214-015-0169-4

DiBraccio, G. A., Luhmann, J. G., Curry, S. M., Espley, J. R., Xu, S., Mitchell, D. L. (2018). The twisted configuration of the Martian magnetotail: MAVEN observations. Geophysical Research Letters, 45(10), 4559-4568. https://doi.org/10.1029/2018GL077251

Dong, Y., Fang, X., Brain, D. A., Hurley, D. M., Halekas, J. S., Espley, J. R., et al. (2019). Magnetic field in the Martian magnetosheath and the application as an IMF clock angle proxy. Journal of Geophysical Research: Space Physics, 124, 4295-4313. https://doi.org/10.1029/2019JA026522

Dong, Y., Fang, X., Brain, D. A., McFadden, J. P., Halekas, J. S., Connerney, J. E., et al. (2015). Strong plume fluxes at Mars observed by MAVEN: An important planetary ion escape channel. Geophysical Research Letters, 42, 8942-8950. https://doi.org/10.1002/2015GL065346

Dubinin, E., Fraenz, M., Fedorov, A., Lundin, R., Edberg, N., Duru, F., \& Vaisberg, O. (2011). Ion energization and escape on Mars and Venus. Space Science Reviews, 162, 173-211. https://doi.org/10.1007/s11214-011-9831-7

Dubinin, E., Fraenz, M., Pätzold, M., McFadden, J., Halekas, J. S., DiBraccio, G. A., et al. (2017). The effect of solar wind variations on the escape of oxygen ions from Mars through different channels: MAVEN observations. Journal of Geophysical Research: Space Physics, 122, 11285-11301. https://doi.org/10.1002/2017JA024741

Dubinin, E., Fraenz, M., Woch, J., Roussos, E., Barabash, S., Lundin, R., et al. (2006). Plasma morphology at Mars: ASPERA-3 Observations, Space Science Reviews, 126, 209-238. https://doi.org/10.1007/s11214-006-9039-4

Dubinin, E., Israelevich, P. L. \& Podgomy, I. M. (1980). Combined magnetosphere, Kosmicheskie Issledovaniia, 18, 470-474. https://doi.org/10.1016/0008-6223(80)90007-X 
Dubinin, E., Lundin, R., Fraenz, M., Woch, J., Barabash, S., Fedorov, A., et al. (2006). Electric Fields within the Martian magnetosphere and ion extraction. ASPERA observations. Icarus, 182(2), 337-342. https://doi.org/10.1016/j.icarus.2005.05.022

Dubinin, E., Modolo, R., Fraenz, M., Pätzold, M., Woch, J., Chai, L., et al. (2019). The induced magnetosphere of Mars: Asymmetrical topology of the magnetic field lines. Geophysical Research Letters, 46. https://doi.org/10.1029/2019g1084387

Dubinin, E., Sauer, K., Lundin, R., Norberg, O., Trotignon, J.-G., Schwingenschuh, K., et al. (1996). Plasma characteristics of the boundary layer in the Martian magnetosphere. Journal of Geophysical Research, 101, 27061-27075. https://doi.org/10.1029/96ja02021

Ergun, R. E., Andersson, L., Fowler, C. M., Woodson, A., Weber, T., Delory, G., et al. (2016). Enhanced $\mathrm{O}_{2}^{+}$loss at Mars due to an ambipolar electric field from electron heating. Journal of Geophysical Research: Space Physics, 121, 4668-4678. https://doi.org/10.1002/2016JA022349

Fang, X., Liemohn, M., Nagy, A., Luhmann, J., \& Ma, Y. (2010). Escape probability of Martian atmospheric ions. Controlling effects of the electromagnetic fields. Journal of Geophysical Research, 115, A04308. https://doi.org/10.1029/2009JA014929

Halekas, J., \& Brain, D. A. (2010). Global distribution, structure, and solar wind control of low-altitude current sheets at Mars. Icarus, 206(1), 64-73. https://doi.org/10.1016/j.icarus.2008.12.032

Halekas, J. S., Brain, D. A., Lillis, R. J., Fillingim, M. O., Mitchell, D. L., \& Lin, R. P. (2006). Current sheets at low altitudes in the Martian magnetotail. Geophysical Research Letters, 33, L13101. https://doi.org/10.1029/2006GL026229

Halekas, J. S., Brain, D. A., Luhmann, J. G., DiBraccio, G. A., Ruhunusiri, S., Harada, Y., et al. (2017). Flows, fields, and forces in the Mars-solar wind interaction. Journal of Geophysical Research: Space Physics, 122(11), 11-320. https://doi.org/10.1002/2017JA024772

Halekas, J., Taylor, E., Dalton, G., Johnson, G., Curtis, D., McFadden, J., et al. (2015). The solar wind ion analyzer for MAVEN. Space Science Reviews, 195, 125-151. https://doi.org/10.1007/s11214-013-0029-z

Hara, T., Brain, D. A., Mitchell, D. L., Luhmann, J. G., Seki, K., Hasegawa, H., \& Jskosky, B. A. (2016). MAVEN observations of a giant ionospheric flux rope near Mars resulting from interaction between the crustal and interplanetary draped magnetic fields. Journal of Geophysical Research: Space Physics, 122, 828-842. https://doi.org/10.1002/2016JA023347

Harada, Y., Halekas, J. S., DiBraccio, G. A., XuEspley, J., McFadden, J. P., Jakosky, B. M., et al. (2018). Magnetic reconnection on dayside crustal magnetic fields at Mars: MAVEN observations. Geophysical Research Letters, 45, 4550-4558. https://doi.org/10.1002/2018GL077281

Harada, Y., Halekas, J. S., McFadden, J. P., Espley, J., DiBraccio, G. A., Mitchell, D. L., et al. (2017). Survey of magnetic reconnection signatures in the Martian magnetotail with MAVEN. Journal of Geophysical Research: Space Physics, 122, 5114-5131. https://doi. org/10.1002/2017JA023952/enleadertwodots

Harada, Y., Halekas, J. S., Xu, S., DiBraccio, G. A., Ruhunishi, S., Hara, T., et al. (2020). Ion jets within current sheets in the Martian magnetosphere. Journal of Geophysical Research: Space Physics, 125, e2020JA028576. https://doi.org/10.1029/2020JA028576

Jakosky, B. M., Lin, R. P., Grebowsky, J. M., Luhmann, J. G., Mitchell, D. F., Beutelschies, G., et al. (2015). The Mars Atmosphere and Volatile Evolution (MAVEN) mission. Space Science Reviews, 195(1-4), 3-48. https://doi.org/10.1007/s11214-015-0139-x

Lavraud, B., \& Larson, D. (2016). Correcting moments of in-situ particle distribution functions for spacecraft electrostatic charging. Journal of Geophysical Research: Space Physics, 121, 8462-8474. https://doi.org/10.1002/2016ja022591

Luhmann, J. G. (1990). A model of the ion wake of Mars. Geophysical Research Letters, 17(6), 869-872. https://doi.org/10.1029/GL017i006p00869

Luhmann, J. G., Elphic, R., Russell, C. T., Mihalov, J. D. \& Wolfe, J. H. (1980). Observations of large scale steady magnetic fields in the dayside Venus ionosphere. Geophysical Research Letters, 7(11), 917-920. https://doi.org/10.1029/g1007i011p00917

Lundin, R., Barabash, S., Homstrom, M., Nilsson, H., Yamauchi, M., Fraenz, M., \& Dubinin, E. (2008). A comet-like escape of ionospheric plasma from Mars. Geophysical Research Letters, 35, L18203. https://doi.org/10.1029/2008g1034811

Mazelle, C., Winterhalter, D., Sauer, K., Trotignon, J. G., Acuña, M. H., Baumgärtel, K., et al. (2004). Bow shock and upstream phenomena at Mars. Space Science Reviews, 111, 115-181. https://doi.org/10.1023/b:spac.0000032717.98679.d0

McFadden, J. P., Kortmann, O., Curtis, D., Dalton, G., Johnson, G., Abiad, R., et al. (2015). MAVEN Supra Thermal and Thermal Ion Compostion (STATIC) instrument. Space Science Reviews, 195, 199-256. https://doi.org/10.1007/s11214-015-0175-6

Moore, K. R., McComas, D. J., Russell, C. T., Mihalov, J. D. (1990). A statistical study of ions and magnetic field in the Venus magnetotail, Journal of Geophysical Research, 95, 12005-12018. https://doi.org/10.1029/ja095ia08p12005

Nagy, A. F., Winterhalter, D., Sauer, K. et al. (2004). The plasma environment of Mars, Space Science Reviews, 111(1), 33-114. https://doi. org/10.1023/b:spac.0000032718.47512.92

Ramstad, R., Brain, D., Dong, Y., Espley, J., Halekas, J., \& Jakosky, B. (2020). The global current systems of the Martian induced magnetosphere. Nature, Astronomy, 4, 979-985. https://doi.org/10.1038/s41550-020-1099-y

Russell, C. T., Mulligan, T., Delva, M., Zhang, T.-L. \& Schwingenschuh, K. (1995). A simple test of the induced nature of the Martian tail. Planetary and Space Science, 43, 875-879. https://doi.org/10.1016/0032-0633(94)00217-f

Russell, C. T. \& Vaisberg, O. (1983). Interaction of the solar wind with Venus. In D. M. Hunten, et al. (Eds.), Venus (pp. 873-940). University of Arizona Press.

Sanchez-Cano, B., Narvaez, C., Lester, M., Mendillo, M., Mayyasi, M., Holmstrom, M., et al. (2020). Mars' ionopause: A matter of pressures. Journal of Geophysical Research: Space Physics, 125, e2020JA028145. https://doi.org/10.1029/2020JA028145

Weber, T., Brain, D., Mitchell, D., Xu, S., Espley, J., Halrkas, J., et al. (2019). Influence of solar wind pressure on Martian crustal magnetic field topology. Geophysical Research Letters, 46, 2347-2354. https://doi.org/10.1029/2019GL081913

Xu, S., Mitchell, D. L., Brain, D. A., Luhmann, J. G., Dong, C., Mazelle, C., et al. (2020). Characterizing Mars's magnetotail topology with respect to the upstream interplanetary magnetic fields. Journal of Geophysical Research: Space Physics, 125, e2019JA027755. https://doi. org/10.1029/2019JA027755

Xu, S., Mitchell, D. L., Liemohn, M., Fang, X., Ma, Y., Luhmann, J. G., et al. (2017). Martian low-altitude magnetic topology deduced from MAVEN/SWEA observations. Journal of Geophysical Research: Space Physics, 122, 1831-1852. https://doi.org/10.1002/2016JA023467

Xu, S., Mitchell, D. L., McFadden, J., Collinson, G., Harada, Y., Lillis, R., et al. (2018). Field-aligned potentials at Mars from MAVEN observations. Geophysical Research Letters, 45, 10119-10127. https://doi.org/10.1029/2018GL080136

Yeroshenko, Y., Riedler, W., Schwingenschuh, K., Luhmann, J. G., Ong, M. \& Russell, C. T. (1990). Magnetotail of Mars: Phobos observations. Geophysical Research Letters, 17, 885-888. https://doi.org/10.1029/g1017i006p00885

Zhang, T. L., Baumjohann, W., Du, J., Nakamura, R., Jarvinen, R., Kallio, E., et al. (2010). Hemispheric asymmetry of the magnetic field wrapping pattern in the Venusian magnetic tail. Geophysical Research Letters, 37, L14202. https://doi.org/10.1029/2010GL044020.201 